\title{
A CLASS OF SCHUR ALGEBRAS
}

BY

M. BRENDER

\begin{abstract}
This paper delineates a class of Schur algebras over a finite group $G$, parametrized by two subgroups $K \triangleleft H \subset G$. The constructed Schur algebra $\mathbf{C}[G]_{K}^{H}$ is maximal for the two properties (a) centralizing the elements of $H$, and (b) containing the elements of $K$ in the identity. Most commonly considered examples of Schur algebras fall into this class.

A complete set of characters of $\mathrm{C} G]_{K}^{H}$ is given in terms of the spherical functions on the group $G$ with respect to the subgroup $H$. Necessary and sufficient conditions are given for this Schur algebra to be commutative, in terms of a condition on restriction multiplicities of characters. This leads to a second-orthogonality-type relation among a subset of the spherical functions. Finally, as an application, a particular Schur algebra of this class is analyzed, and shown to be a direct sum of centralizer rings.
\end{abstract}

1. Introduction. The purpose of this paper is to delineate a particular class of Schur algebras inside $\mathbf{C}[G]$, where $G$ is a finite group and $\mathbf{C}$ is the complex field. The algebras are parametrized by two subgroups of $G$, one normal inside the other. For various choices of the subgroups, the Schur algebras in question become ones that have been studied by various authors. It is hoped that this work will unify diverse results and point toward further clarification. In this section we quote the relevant definitions, results, and notation to be used.

We take as the definition of Schur ring that given by Tamaschke [5]: $T \subset \mathbf{Z}[G]$ is called a Schur ring over $G$ if:

(1) $T$ is a ring (not necessarily with unit), and

(2) $T$ has a $\mathbf{Z}$-basis $\left\{\tau_{1}, \ldots, \tau_{s}\right\}$ where each $\tau_{i}$ is a simple sum of elements of the finite group $G$, so that $\tau_{i}=\Sigma_{g \in T_{i}} g$ where the subsets $T_{i} \subset G$ satisfy the following:

(a) $T_{i} \cap T_{j}=\varnothing$ if $i \neq j$,

(b) $\cup_{i} T_{i}=G$, and

(c) For each $i,\left(T_{i}\right)^{-1}=T_{j}$ for some $j$.

We call the subsets $T_{i}$ the $T$-classes, and number them so that $1 \in T_{1}$.

If $T$ is a Schur ring, the associated Schur algebra $C T$ is the subalgebra of C $[G]$ spanned by $\left\{\tau_{1}, \ldots, \tau_{s}\right\}$. CT may be viewed as the set of elements of

Received by the editors February 7, 1977.

AMS (MOS) subject classifications (1970). Primary 20C05, 20C15, 16A64.

Key words and phrases. Schur algebra, $S$-ring, semisimple algebra, finite group, spherical function, character, double coset algebra, centralizer ring. 
C $[G]$ whose coefficients are constant along each of the subsets $T_{i}$. These algebras were studied by Wielandt [7], [8], although he calls them $S$-rings. In [7] he proves that $\tau_{1} / T_{1}$ is the unit element of $\mathbf{C} T$ and in an oft-cited footnote (p. 386) that $\mathbf{C} T$ is a semisimple algebra.

Examples of Schur algebras include the following:

(1) The group algebra $\mathbf{C}[G]$ itself.

(2) The center $\mathbf{C}[G]^{G}$ of the group algebra, for which the partition of $G$ is into conjugacy classes.

(3) The centralizer in $\mathbf{C}[G]$ of a subgroup $H$, denoted $\mathrm{C}[G]^{H}$. Here the $T$-classes are the $H$-conjugacy classes of $G$. This algebra has been studied by Travis [6] and Karlof [2].

(4) The double coset algebra $\mathbf{C}[H \backslash G / H]$ for which $G$ is partitioned into its $H$ - $H$ double cosets. This algebra was considered by Tamaschke [4], and Karlof [3] has shown that every $\boldsymbol{H}$-class Schur algebra is isomorphic to a particular double coset Schur algebra.

The class of Schur algebras defined in $\$ 2$ includes these examples as special cases. We employ the following notations and results.

$G$ will be any finite group, and $H$ any subgroup. Let $\hat{G}$ and $\hat{H}$ be the sets of complex irreducible characters of $G$ and $H$ respectively. For $\chi \in \hat{G}$ and $\psi \in \hat{H}$, let $c_{\chi \psi}$ be the multiplicity with which $\psi$ is contained in the restriction of $\chi$ to $H$, and let $f_{x}$ and $f_{\psi}$ be the degrees of $\chi$ and $\psi$.

Given $\chi \in \hat{G}$ and $\psi \in \hat{H}$, Travis has defined the associated spherical function on $G$ by

$$
\varphi_{\chi \psi}(g)=\frac{1}{|H|} \sum_{h \in H} \chi(g h) \psi\left(h^{-1}\right)
$$

and has shown that these spherical functions have the following properties:

(1) $\varphi_{\chi \psi}$ is constant along $H$-conjugacy classes of $G$, and is a nonzero function if and only if $c_{x \psi} \neq 0$.

(2) $\left(\varphi_{\chi \psi}, \varphi_{\chi^{\prime} \psi^{\prime}}\right)_{G}=|G|^{-1} \Sigma_{g \in G} \varphi_{x \psi}(g) \overline{\varphi_{x^{\prime} \psi^{\prime}}(g)}=\delta_{x x^{\prime}} \delta_{\psi \psi^{\prime}} c_{\chi \psi} / f_{\chi} f_{\psi}$.

(3) The nonzero $\varphi_{x \psi}$ 's are consequently linearly independent.

(4) $\left.\varphi_{\chi \psi}\right|_{H}=c_{\chi \psi} \psi / f_{\psi}$.

If we extend $\varphi_{x \psi}$ linearly to all of $\mathbf{C}[G]$, and then restrict to $\mathrm{C}[G]^{H}$, we can view $\varphi_{\chi \psi}$ as a function on $\mathbf{C}[G]^{H}$. We now quote:

ThEOREM 1.1 (TRAVIS). The set $\left\{\varphi_{x \psi} \mid c_{x \psi} \neq 0\right\}$ is a complete set of irreducible complex characters of the semisimple algebra $\mathrm{C}[G]^{H}$.

It will be convenient to have the following lemma set down, although it is certainly well known in the folklore. I would like to thank Pat Gallagher for his suggestion to state certain of the claims in the language of ideals. 
Lemma 1.2. Let $I \neq 0$ be a two sided ideal of a semisimple algebra $A$. Then each irreducible character of $A$ on restriction to I either vanishes or gives an irreducible character of I. Moreover, no two irreducible characters of $A$ restrict to the same irreducible character of I, and all irreducible characters of I arise by restricting some character of $A$.

Proof. This lemma is similar to Theorem 1.1 of [1] and can be proved using similar arguments. We prefer, however, to consider regular representations.

Let $A=\bigoplus_{i=1}^{s} R_{i}$ be the decomposition of $A$ into simple components, where each $R_{i}$ is the sum of all minimal left ideals upon which $A$ acts by left multiplication with irreducible character $\chi_{i}$. If $1=e_{1}+\cdots+e_{s}$ is the decomposition of 1 into orthogonal central idempotents then $I=\bigoplus_{i=1}^{s} e_{i} I e_{i}$. By simplicity, each $e_{i} I e_{i}$ is either 0 or all of $R_{i}$. Hence $I=\bigoplus_{i \in J} R_{i}$ for a particular subset $J \subset\{1, \ldots, s\}$.

On each submodule $R_{i}, A$ acts with character $f_{i} \chi_{i}$ where $f_{i}$ is the degree of $\chi_{i}$. Now restrict the operators to $I$. If $i \notin J, I$ annihilates $R_{i}$ and so $\left.f_{i} \chi_{i}\right|_{I}=0$. If $i \in J, I$ contains the full ring of endomorphisms of each minimal ideal $L \subset R_{i}$ and so $\left.f_{i} \chi_{i}\right|_{I}$ is still the sum of $f_{i}$ irreducible representations. Hence if $\left.\chi_{\mathrm{i}}\right|_{I}$ does not vanish, it is irreducible.

Now suppose neither $\chi_{i}$ nor $\chi_{j}$ vanishes on $I$, with $i \neq j$. Then since $e_{i}$ annihilates $R_{j}$, we have $\chi_{j}\left(e_{i}\right)=0$ but $\chi_{i}\left(e_{i}\right)=\operatorname{dim}\left(L_{i}\right)$ for any minimal left ideal $L_{i} \subset R_{i}$. Hence $\chi_{i}$ and $\chi_{j}$ differ on $I$.

For the last claim, start with any irreducible character $\chi$ of $I$. Then $\chi$ must be afforded by some minimal left ideal $L \subset I$. By minimality, $L \subset R_{i}$ for some $i \in J$. But then $\chi=\chi_{i}$ on $I$.

2. The Schur algebras $\mathrm{C}[G]_{K}^{H}$. Let $H \subset G$ be finite groups, and let $K \triangleleft H$. Denote by $H / K$ the characters of $H$ whose kernels contain $K$.

Proposition 2.1. $K \rtimes H$ acts on $G$ via $(k, h) g=k h g h^{-1}$. The number of orbits is

$$
\sum_{\psi \in H \hat{\gamma} K}\left(\psi^{G}, \psi^{G}\right)_{G}=\sum_{\substack{x \in \hat{G} \\ \psi \in H / K}}\left(c_{x \psi}\right)^{2} .
$$

Proof. That we have a group action is immediately verified from the group law in $K \rtimes H$. Since $\psi^{G}=\Sigma_{\chi \in \hat{G}} c_{\chi \psi} \chi$, the two formulae are clearly equal. It remains to show that they count the orbits. Begin by noting that by Frobenius reciprocity, the first of the two equals 


$$
\begin{aligned}
\sum_{\psi \in H \hat{\gamma} K}\left(\left.\psi^{G}\right|_{H}, \psi\right)_{H} & =\sum_{\psi \in H\lceil K} \frac{1}{|H|} \sum_{h \in H} \psi^{G}(h) \psi\left(h^{-1}\right) \\
& =\sum_{\psi \in H \uparrow K} \frac{1}{|H|^{2}} \sum_{\substack{h \in H \\
g \in G}} \check{\psi}\left(g h g^{-1}\right) \psi\left(h^{-1}\right)
\end{aligned}
$$

where $\check{\psi}$ is defined to equal $\psi$ on $H$ and vanish off $H$. Using the multiplication formula for characters, we rewrite the last sum as

$$
\sum_{\psi \in H \hat{\gamma} K} \frac{1}{|H|^{3}} \sum_{\substack{\lambda, h \in H \\ g \in G}} f_{\psi} \check{\psi}\left(g^{\prime} g^{-1} \lambda h^{-1} \lambda^{-1}\right) .
$$

Reversing the order of summation, we have

$$
\frac{1}{|H|^{3}} \sum_{\substack{\lambda, h \in H \\ g \in G}} \sum_{\psi \in H / K} f_{\psi} \check{\psi}\left(g h g^{-1} \lambda h^{-1} \lambda^{-1}\right)=\frac{1}{|H|^{3}} \sum(H: K)
$$

where the last sum is taken over all triples $(\lambda, h, g)$ in $H \times H \times G$ such that ghg ${ }^{-1} \lambda h^{-1} \lambda^{-1} \in K$. Introducing a new summation variable $k \in K$, we rewrite the sum as $\left(|H|^{2}|K|\right)^{-1} \Sigma 1$ in which we now sum over all quadruples $(k, \lambda, h, g)$ in $K \times H \times H \times G$ such that $g h g^{-1} \lambda h^{-1} \lambda^{-1}=k$. Since this is equivalent to $\left(g \lambda^{-1}\right)\left(\lambda h \lambda^{-1}\right)\left(g \lambda^{-1}\right)^{-1}\left(\lambda h \lambda^{-1}\right)=k$, we make the changes of variables $g$ for $g \lambda^{-1}$ and $h$ for $\lambda h \lambda^{-1}$, and sum over quadruples satisfying $g h g^{-1} h^{-1}=k$. Hence our sum is simply

$$
\frac{1}{|H|^{2}|K|} \sum 1=\frac{1}{|H||K|} \sum 1
$$

where the sum on the $L H S$ is over all quadruples $(k, \lambda, h, g)$ such that $g=k h g h^{-1}$ and the sum on the RHS is over pairs $(k, h) \in K \rtimes H, g \in G$, $(k, h) g=g$, which gives the number of orbits by the Burnside orbit formula.

If two elements $g$ and $g^{\prime}$ of $G$ are in the same orbit under $K \rtimes H$ we will write $g \sim g^{\prime}$ and we will use $(g)$ to denote the orbit containing $g$. Choose a complete set of orbit representatives $\left\{g_{1}=1, g_{2}, \ldots, g_{s}\right\}$ so that as a set $G$ is the disjoint union $\cup_{i=1}^{s}\left(g_{i}\right)$.

The following observations follow immediately from the action of $K \rtimes H$ on $G:\left(g_{1}\right)=(1)=K ; k\left(g_{i}\right)=\left(g_{i}\right)$ for any $k \in K$; and $h\left(g_{i}\right) h^{-1}=\left(g_{i}\right)$ for any $h \in H$. Hence each orbit $\left(g_{i}\right)$ is a union of right cosets of $K$ in $G$, and at the same time, a union of $H$-conjugacy classes in $G$. Moreover, given any partition $G=\bigcup_{j=1}^{t} S_{j}$ (disjoint) with the property that each $S_{j}$ is invariant under left multiplication by each element of $K$, and also under conjugation by each element of $H$, it is clear that each $S_{j}$ is a union of some of the orbits $\left(g_{i}\right)$.

For each $i=1, \ldots, s$ define $\tau_{i}=\Sigma_{g \in\left(g_{i}\right)} g$. Translating the above remarks to the group algebra yields the following: 
Lemma 2.2. For all $i$, all $k \in K$, all $h \in H$ we have $k \tau_{i}=h \tau_{i} h^{-1}=\tau_{i}$. If $x \in \mathbf{C}[G]$ satisfies $k x=x$ for all $k \in K$ and $h x h^{-1}=x$ for all $h \in H$, then $x$ is a linear combination of the $\tau_{i}^{\prime}$ 's.

Proposition 2.3. The subset $T$ of $\mathbf{Z}[G]$ spanned by the $\tau_{i}$ 's is a Schur ring. The corresponding Schur algebra $\mathbf{C T}$ satisfies the following two properties:

(a) $\mathrm{CT}$ centralizes the elements of $\mathrm{H}$.

(b) The elements of $K$ all appear in the identity element of $\mathbf{C T}$.

Moreover, every Schur algebra over G satisfying (a) and (b) above is a Schur subalgebra of $\mathbf{C} T$.

Proof. Clearly $T$ is a $\mathbf{Z}$-module. To show $T$ is a ring we show $\tau_{i} \tau_{j} \in T$ for all $i$ and $j$. Note that for any $k \in K$ and any $h \in H, k\left(\tau_{i} \tau_{j}\right)=\left(k \tau_{i}\right) \tau_{j}=\tau_{i} \tau_{j}$ and $h \tau_{i} \tau_{j} h^{-1}=h \tau_{i} h^{-1} h \tau_{j} h^{-1}=\tau_{i} \tau_{j}$ and so by Lemma $2.2, \tau_{i} \tau_{j}$ is a linear combination of the $\tau$ 's. Since the coefficients must obviously be integers, $\tau_{i} \tau_{j} \in T$. For the first claim, it only remains to show that for each $i$, $\left(g_{i}\right)^{-1}=\left(g_{j}\right)$ for some $j$. Since it is easily shown that $g \sim g^{\prime}$ if and only if $g^{-1} \sim g^{-1}$, the claim is proved.

That $\mathbf{C T}$ satisfies (a) follows immediately from Lemma 2.2, and (b) is clear since the identity element of $\mathbf{C} T$ is $\tau_{1} / K$. Lastly, suppose $\mathrm{CS}$ is a Schur algebra over $G$ satisfying (a) and (b), with simple basis elements $\sigma_{1}, \ldots, \sigma_{t}$. Wielandt shows [7] that the elements appearing in $\sigma_{1}$ form a subgroup, and that the identity element of $\mathbf{C S}$ is just $\sigma_{1}$ divided by a scalar. This together with assumption (b) guarantees that if $x \in \mathbf{C S}, k x=x$ for each $k \in K$. By assumption (a), we have $h x h^{-1}=x$ for each $h \in H$. Hence by the lemma, $x \in \mathbf{C} T$.

The Schur algebra $\mathbf{C} T$ of the proposition will be denoted $\mathbf{C}[G]_{K}^{H}$, and is the object of this paper. We begin by stating

Proposition 2.4. Let $K^{\prime} \subset K$ be two normal subgroups of $H$. Then $\mathbf{C}[G]_{K}^{H}$ is a two-sided ideal of $\mathbf{C}[G]_{K^{\prime}}^{H}$.

Proof. Since $\mathbf{C}[G]_{K}^{H}$ centralizes $H$, and since the elements of $K^{\prime}$ all appear in the identity of $\mathbf{C}[G]_{K}^{H}$, by the last part of Proposition $2.3, \mathbf{C}[G]_{K}^{H}$ is a subalgebra of $\mathbf{C}[G]_{K^{\prime}}^{H}$.

Let $\tau_{1}, \ldots, \tau_{s}$ be the simple basis elements of $\mathbf{C}[G]_{K}^{H}$ and $\sigma_{1}, \ldots, \sigma_{t}$ those of $\mathbf{C}[G]_{K^{\prime}}^{H}$. To complete the proof, it will suffice to show that $\tau_{i} \sigma_{j}$ and $\sigma_{j} \tau_{i}$ always lie in $\mathbf{C}[G]_{K}^{H}$. This is easily done using the second part of Lemma 2.2 .

In particular, for any $K \triangleleft H$, we have that $\mathbf{C}[G]_{K}^{H}$ is a two-sided ideal of $\mathbf{C}[G]_{1}^{H}$. Since $\mathbf{C}[G]_{1}^{H}=\mathbf{C}[G]^{H}$ we are now in a position to apply Travis' results on the irreducible representations of $\mathrm{C}[G]^{H}$ to determine the irreducible characters of $\mathrm{C}[G]_{K}^{H}$. 
3. The characters of $\mathrm{C}[G]_{K}^{H}$. To obtain a complete set of characters of $\mathrm{C}[G]_{K}^{H}$, we can, in the light of Lemma 1.2, Proposition 2.4, and Travis' description of the characters of $\mathbf{C}[G]^{H}$, simply restrict the spherical functions $\varphi_{X \psi}$ to $\mathbf{C}[G]_{K}^{H}$.

Proposition 3.1. If $\psi \in H \hat{/ K}$, then $\varphi_{x \psi}(g)=\varphi_{x \psi}((k, h) g)$ for all $(k, h) \in$ $K \rtimes H$, and all $g \in G$.

Proof. $\varphi_{x \psi}((k, h) g)=\varphi_{x \psi}\left(k h g h^{-1}\right)=\varphi_{x \psi}\left(g h^{-1} k h\right)$ since all the spherical functions are constant along $H$-conjugacy classes. Hence

$$
\varphi_{x \psi}((k, h) g)=\frac{1}{|H|} \sum_{r \in H} \chi(g r) \psi\left(r^{-1} h^{-1} k h\right) .
$$

Letting $R$ be a representation of $H$ affording $\psi$, we have

$$
\frac{1}{|H|} \sum_{r \in H} \chi(g r) \operatorname{Tr}\left(R\left(r^{-1}\right) R\left(h^{-1} k h\right)\right)=\frac{1}{|H|} \sum_{r \in H} \chi(g r) \psi\left(r^{-1}\right)
$$

which is just $\varphi_{x \psi}(g)$. Hence each such $\varphi_{\chi \psi}$ assumes constant values along $T$-classes.

Proposition 3.2. The set $\left\{\varphi_{x \psi} \mid \psi \in H \hat{\gamma} K, c_{x \psi} \neq 0\right\}$, viewed as a set of functions on $\mathbf{C}[G]_{K}^{H}$, gives a complete set of irreducible characters.

Proof. By the preceding proposition, these spherical functions do not vanish on restriction to $\mathrm{C}[G]_{K}^{H}$. By Lemma 1.2 , we therefore have a set of distinct irreducible characters. Since the identity of $\mathrm{C}[G]_{K}^{H}$ is $\tau_{1} / K$, the dimension of the representation space affording any of these $\varphi_{x \psi}$ is

$$
\varphi_{\chi \psi}\left(\tau_{1} /|K|\right)=(1 /|K|)|K| \varphi_{\chi \psi}(1)=c_{\chi \psi} .
$$

By Proposition 2.1 we have that the dimension over $\mathbf{C}$ of $\mathbf{C}[G]_{K}^{H}$ is $\sum_{\psi \in H \hat{T} ; \chi x}\left(c_{\chi \psi}\right)^{2}$.

Since the sum of the squares of the degrees of all irreducible representations of any semisimple algebra is the dimension of the algebra, we must already have them all.

Corollary 3.3. If $\psi \notin H \hat{/ K}$, then $\varphi_{X \psi}$ vanishes on $\mathrm{C}[G]_{K}^{H}$.

Proof. This follows immediately from Proposition 3.2 and Lemma 1.2.

4. Multiplicity-free cases. The subgroup $H \subset G$ has been called multiplicity-free if for all $\chi \in \hat{G}$ and $\psi \in \hat{H}, c_{\chi \psi}=0$ or 1 . We define the following, less restrictive property: If $K \triangleleft H$, we call $H \subset G$ multiplicity-free on $K$ if for all $\chi \in \hat{G}$, and for all $\psi \in H \hat{\mid K}, c_{\chi \psi}=0$ or 1 . It is obvious that if $K^{\prime} \subset K$, with $K^{\prime}$ and $K$ both normal in $H$, that $H \subset G$ multiplicity-free on $K^{\prime}$ implies $H \subset G$ multiplicity-free on $K$.

It has been shown [6] that $\mathrm{C}[G]^{H}$ is commutative if and only if $H \subset G$ is 
multiplicity-free, and that under these circumstances, the $\varphi_{x \psi}$ 's span all the $H$-class functions on $G$. We now generalize these results by examining the conditions under which $\mathrm{C}[G]_{K}^{H}$ is commutative.

Proposition 4.1. The following conditions are equivalent:

(a) $\mathbf{C}[G]_{K}^{H}$ is a commutative Schur algebra.

(b) $H \subset G$ is multiplicity-free on $K$.

(c) The set $\left\{\varphi_{x \psi} \mid \psi \in H / K\right\}$ spans the space of all T-class functions.

Proof. Any semisimple algebra is commutative if and only if all its irreducible representations have degree 1 . Since the degrees of the irreducible representations here are the $c_{\chi \psi}$, we have the equivalence of (a) and (b).

As with any function on $G, \varphi_{x \psi}$ may be seen as an element of $\mathrm{C}[G]$ by taking $\varphi_{x \psi}=\Sigma_{g} \varphi_{x \psi}(g) g$. By Proposition 3.1 the elements $\varphi_{x \psi}$ with $\psi \in$ $H \hat{/ K}$ lie inside $\mathbf{C}[G]_{K}^{H}$. Viewing $\mathbf{C}[G]_{K}^{H}$ as a vector subspace of $\mathbf{C}[G]$, we have that the vectors in $B=\left\{\varphi_{\chi \psi} \mid \psi \in H / K\right\}$ are linearly independent and so form a basis for a subspace $S$ of $\mathbf{C}[G]_{K}^{H}$. But

$$
\operatorname{dim} \mathbf{C}[G]_{K}^{H}=\sum_{\substack{\psi \in H \hat{/ K} \\ x \in \hat{G}}}\left(c_{\chi \psi}\right)^{2} \geqslant \sum_{\substack{\psi \in H / K \\ x \in \hat{G}}} 1=\operatorname{dim} S
$$

with equality if and only if (b) holds.

But we will have equality if and only if $S$ is all of $\mathrm{C}[G]_{K}^{H}$, hence the equivalence of (b) and (c).

The original basis for the space $\mathbf{C}[G]_{K}^{H}$ was the set of $\tau_{i}$ (the characteristic functions on the $T$-classes). We can now give the matrices of transformation between these two bases:

Proposition 4.2. If $H \subset G$ is multiplicity-free on $K$, we have

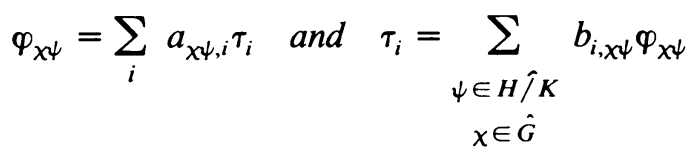

where

$$
a_{\chi \psi, i}=\frac{1}{\left|\tau_{i}\right|} \varphi_{\chi \psi}\left(\tau_{i}\right) \quad \text { and } \quad b_{i, \chi \psi}=\frac{f_{\chi} f_{\psi}}{|G|} \overline{\varphi_{\chi \psi}\left(\tau_{i}\right)} \text {. }
$$

Proof. Since $\varphi_{\chi \psi}\left(\tau_{i}\right)=\left|\tau_{i}\right| \varphi_{\chi \psi}(g)$ for each $g \in\left(g_{i}\right)$ the value for $a_{\chi \psi, \mathrm{i}}$ is clear.

Taking the dot product of the second sum with $\varphi_{\chi_{1} \psi_{1}}$, we have

$$
\left(\tau_{i}, \varphi_{\chi_{1} \psi_{1}}\right)_{G}=b_{i, x_{1} \psi_{1}} \frac{c_{\chi_{1} \psi_{1}}}{f_{\chi_{1}} f_{\psi_{1}}}=\frac{b_{i, \chi_{1} \psi_{1}}}{f_{\chi_{1}} f_{\psi_{1}}}
$$


and so

$$
b_{i, \chi_{1} \psi_{1}}=f_{\chi_{1}} f_{\psi_{1}} \frac{1}{|G|} \sum_{g \in\left(g_{i}\right)} \overline{\varphi_{\chi_{1} \psi_{1}}(g)}
$$

and the result follows.

It should be noted that according to the second part of the proposition,

$$
\tau_{i}=\sum_{\substack{\psi \in H \hat{\gamma} K \\ x \in \hat{G}}} \frac{f_{\chi} f_{\psi}}{|G|} \overline{\varphi_{\chi \psi}\left(\tau_{i}\right)} \varphi_{\chi \psi} .
$$

Adding up the coefficients of each $g \in\left(g_{j}\right)$ on either side leads to

$$
\delta_{i j}\left|\tau_{i}\right|=\sum_{\substack{\psi \in H / K \\ x \in \hat{G}}} \frac{f_{\chi} f_{\psi}}{|G|} \overline{\varphi_{\chi \psi}}\left(\tau_{i}\right) \varphi_{\chi \psi}\left(\tau_{j}\right)
$$

or

$$
\frac{1}{|G|} \sum_{\substack{\psi \in H \hat{\jmath} K \\ x \in \hat{G}}} \frac{f_{\chi} f_{\psi}}{\left|\tau_{i}\right|} \overline{\varphi_{x \psi}\left(\tau_{i}\right)} \varphi_{x \psi}\left(\tau_{j}\right)=\delta_{i j}
$$

which is a second-orthogonality relation among the spherical functions with $\psi \in H \hat{/ K}$, under the assumption that $H \subset G$ is multiplicity-free on $K$.

5. Applications. Each of the examples of Schur algebras mentioned in $\S 1$ is realized with the proper choice of $K$ and $H$.

(1) $\mathbf{C}[G]=\mathbf{C}[G]_{1}^{1}$,

(2) $\mathbf{C}[G]^{G}=\mathbf{C}[G]_{1}^{G}$,

(3) $\mathbf{C}[G]^{H}=\mathbf{C}[G]_{1}^{H}$,

(4) The double coset algebra $\mathbf{C}[H \backslash G / H]$ is simply $\mathbf{C}[G]_{H}^{H}$. By Proposition 2.1 we have that the number of $H \backslash G / H$ double cosets is $\left(1_{H}^{G}, 1_{H}^{G}\right)_{G}$. The characters of $\mathbf{C}[H \backslash G / H]$ are the so-called zonal spherical functions, those of form $\varphi_{x 1}$.

For another example, choose $K$ to be $H^{\prime}$, the commutator subgroup of $H$. Then $H / H^{\prime}$ is simply the set of one-dimensional characters of $H$. By Proposition 3.2, the characters of $\mathrm{C}[G]_{H^{\prime}}^{H}$ are the $\varphi_{\chi \psi}$ satisfying $f_{\psi}=1$.

Curtis and Fossum [1] consider the following situation: They start with $\eta$, a linear character of $H$, and the primitive idempotent $e=|H|^{-1} \sum_{h \in H} \eta\left(h^{-1}\right) h$ in $\mathbf{C}[H]$ that generates the minimal ideal affording $\eta$ (actually they work over a general splitting field, but $\mathbf{C}$ will serve our purpose). They then study the structure of the centralizer ring $e \mathbf{C}[G] e$. For the various linear characters $\eta \in H \hat{/ H^{\prime}}$ the relationship between $e \mathbf{C}[G] e$ and $\mathbf{C}[G]_{H^{\prime}}^{H}$ is given by 
Proposition 5.1. The ring e $\mathrm{C}[G]$ e is a two-sided ideal of $\mathbf{C}[G]_{H^{\prime}}^{H}$. Moreover, if we write $\mathrm{C}[G]_{H^{\prime}}^{H}=\sum_{\psi \in H / H^{\prime}}, \Sigma_{\chi} R_{x \psi}$ where $R_{x \psi}$ is the simple component corresponding to the character $\varphi_{\chi \psi}$, then eC[G]e = $\Sigma_{\chi} R_{x}$.

Proof. Since $\eta$ is linear, for any $h \in H$, he $=e h=\eta(h) e$. If $\alpha \in \mathbf{C}[G]$, it follows then that keae $=\eta(k) e \alpha e=e \alpha e$ and heaeh $h^{-1}=\eta(h) e \alpha \eta\left(h^{-1}\right) e=$ $e \alpha e$ for every choice of $h \in H$ and $k \in H^{\prime}$. Hence by Lemma 2.2,eC[G]e is contained in $\mathrm{C}[G]_{H^{\prime}}^{H}$.

Since $\mathbf{C}[G]_{H^{\prime}}^{H} \subset \mathbf{C}[G]^{H}$, to show $e \mathbf{C}[G] e$ is a left ideal of $\mathbf{C}[G]_{H^{\prime}}^{H}$, it will suffice to show that $b e \mathrm{C}[G] e \subset e \mathrm{C}[G] e$ for each simple basis element $b$ of $\mathrm{C}[G]^{H}$. Hence it will suffice to show $\left(\sum_{h \in H} h g h^{-1}\right) e \alpha e \subset e \mathbf{C}[G] e$ for all $\alpha \in \mathbf{C}[G]$ and any $g \in G$. We have

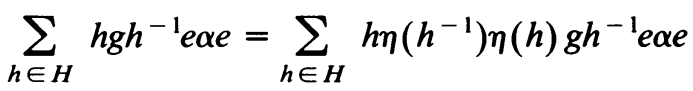

$$
\begin{aligned}
& =\sum_{h \in H} \eta\left(h^{-1}\right) h g \eta(h) h^{-1} \text { eqe. }
\end{aligned}
$$

Since $\eta(h) h^{-1} e=e$ this is

$$
\sum_{h \in H} \eta\left(h^{-1}\right) \text { hgeae }=|H| \text { egeae } \in e \mathbf{C}[G] e .
$$

Arguing symmetrically on the right yields that $e \mathbf{C}[G] e$ is a two-sided ideal of $\mathrm{C}[G]_{H^{\prime}}^{H}$.

Now take any character of $\mathbf{C}[G]_{H}^{H}$, i.e. choose any $\varphi_{x \psi}$ with $f_{\psi}=1$. As the proof of Lemma 1.1 shows, the simple component $R_{\chi \psi}$ of $\mathrm{C}[G]_{H^{\prime}}^{H}$ associated with $\varphi_{x \psi}$ is or is not a summand of $e \mathbf{C}[G] e$ according as $\varphi_{x \psi}$ does not or does vanish on $e \mathbf{C}[G] e$. But

$$
\begin{aligned}
\varphi_{x \psi}(e) & =\frac{1}{|H|} \sum_{h \in H} \eta\left(h^{-1}\right) \varphi_{x \psi}(h) \\
& =\frac{1}{|H|} \sum_{h \in H} \eta\left(h^{-1}\right) c_{x \psi} \frac{\psi(h)}{f_{\psi}}=c_{x \psi} \delta_{\psi \eta},
\end{aligned}
$$

and so the various $\varphi_{x \eta}$ do not vanish on $e \mathrm{C}[G] e$. Further, if $\psi \neq \eta, \varphi_{x \psi}(e)=$ 0 . Since $e$ is the unit of $e \mathrm{C}[G] e$, it follows that these $\varphi_{x \psi}$ vanish on $e \mathbf{C}[G] e$ and the claim is proved.

\section{REFERENCES}

1. C. W. Curtis and T. V. Fossum, On centralizer rings and characters of representations of finite groups, Math. Z. 107 (1968), 402-406.

2. J. Karlof, The subclass algebra associated with a finite group and subgroup, Trans. Amer. Math. Soc. 207 (1975), 329-341.

3. , The subclass algebra associated with a finite group and subgroup. II (to appear).

4. O. Tamaschke, $S$-rings and the irreducible representations of finite groups, J. Algebra 1 (1964), 215-232. 
5. (1970), 340-360.

6. D. Travis, Spherical functions on finite groups, J. Algebra 29 (1974), 65-76.

7. H. Wielandt, Zur theorie der einfach transitiven Permutationsgruppen. II, Math. Z. 52 (1949), 384-393.

8. Finite permutation groups, Academic Press, New York and London, 1964.

Current address: 609 West 114 Street, New York, New York 10025 